\title{
Analysis of Quality of Service for the AOMDV Routing Protocol
}

\author{
Vivek B. Kute. \\ Comp. Engg. Deptt \\ St. Vincent Pallotti College of Engg \& Tech, \\ Nagpur, India \\ vivek_kute@rediffmail.com
}

\author{
M. U. Kharat \\ Comp. Engg Deptt. \\ MET's Institute of Engg, \\ Nashik, India \\ mukharat@rediffmail.com
}

\begin{abstract}
Due to the dynamic nature of Mobile Ad-hoc Networks (MANETs), the provision of Quality of Service (QoS) guarantees is challenging. The route failure probability in a MANET is increased due to the mobility of nodes, which increases routing overhead. Multi-path routing protocols have relatively greater ability to reduce the routing overheads. This paper discuses the performance analysis of the Ad-hoc On-Demand Multi-Path Distance Vector (AOMDV) routing protocol. AOMDV is a multipath extension of a very well known single path routing protocol, (AODV). Extensive simulations were carried out using ns-2.34 and the study concluded that for CBR traffic AOMDV performance degrades as the data packet generation rate increases.
\end{abstract}

Keywords-Ad-hoc; mobile; network; MANET; AOMDV; QoS;

\section{INTRODUCTION}

A mobile Ad-hoc network is a self configuring network of mobile devices. The provision of Quality of Service (QoS) guarantees is much more challenging in Mobile Ad-hoc Networks (MANETs) than that of wire-line networks. This is mainly due to the mobile nature of MANETs' nodes, and other characteristics of MANETs such as Multi hop communication and lack of central coordination. Therefore, designing a MANET routing protocol that guarantees the desired QoS is challenging.

Many routing protocols for MANETs have been proposed. Depending on the time of route discovery MANET routing protocols are divided into two categories; table-driven (proactive) routing protocol and on-demand (reactive) routing protocol. In table-driven routing protocols the routes are discovered and refreshed periodically. All routing related information is stored in routing tables at each node. Whenever a traffic source needs a route, it uses the route available in the routing table. In contrast to this, the traffic source initiates route discovery process when it needs route in case of on-demand routing protocols.

MANET nodes are mobile and, hence, route failure probability is greater. The route discovery process has to start whenever a route fails. Each route discovery flood is associated with significant latency and overhead.
Among the on-demand routing protocols, MANET multipath routing protocols have relatively greater ability to reduce the route discovery frequency than MANET single path routing protocols. On-demand multi-path routing protocols discover multiple paths between a source-destination pair, in a single route discovery. So a new discovery is needed only when all these paths fail. In contrast, a single path routing protocol has to invoke a new route discovery whenever the only path from source to destination fails. [1]

The Ad-hoc On-demand Multi-path Distance Vector (AOMDV) routing protocol is a multi-path extension of the Ad-hoc On-demand Distance Vector (AODV) routing protocol. AOMDV has three novel aspects compared to other on-demand multi-path routing protocols. Firstly, it does not have internodal coordination overheads like some other protocols. Secondly, it ensures disjoint ness of alternate routes via distributed computation without the use of source routing. Thirdly, AOMDV computes alternate paths with minimal additional overhead over AODV. It does this by exploiting as much as possible already available alternate path routing information [1].

In this paper we analyze the performance of the AOMDV routing protocol with two different traffic types, CBR and TCP. The rest of this paper is organized as follows. In section 2 we discuss different QoS issues for MANETs. We review the AOMDV routing protocol in section 3. In section 4 we present performance evaluation of AOMDV's CBR and TCP traffic using network simulator-2. Finally in section 5 we present the conclusion.

\section{QOS ISSUES FOR MANETS}

Multimedia applications often have stringent time and reliability-sensitive service requirements, which the network must cater to. Networks are expected to provide guaranteed QoS. The traditional best effort delivery network can not guarantee today's requirements. The majority of the solutions proposed in MANET have focused on the two metrics, throughput and delay. 
Providing better QoS in MANET is challenging due to the following issues:

- Node Mobility: MANET nodes move at will. This makes the topology dynamic. This means that topology information has a limited lifetime and must be updated frequently to allow data packets to be routed to their destinations. This updating means more routing overheads. Also, due to the node mobility packet losses increase, the end to end delay gets also affected.

- Lack of Central Control: The principal advantage of MANET is that it is deployed without planning in unknown terrains, hazardous conditions and its members can change dynamically. This makes it difficult to have any centralized control. Hence the controlling activities will be distributed among the nodes, which require lot of information exchange. This also adds up to the routing overheads. [2, 3].

Designing a routing protocol to meet these QoS demands is challenging. In [4, 5] AODV routing protocol is analyzed for performance in the view of meeting different QoS demands. In [2], it was shown that multi-path routing performs better than single path routing. It was also shown that AOMDV offers a significant reduction in delay and routing load. The impact of traffic flow on energy consumption of AOMDV compared with AODV is analyzed in [6]. The authors proposed load spreading on different paths, so that the load will be balanced in more paths resulting in energy preservation of nodes in the network. AODV and AOMDV were compared in presence of selfish behaviors of nodes in [7]. It was shown that multi-path routing is better than single path routing in presence of selfish behavior.

\section{AOMDV ROUTING PROTOCOL}

The basic idea behind multi-path routing is of finding multiple paths between a source and a destination. On-demand routing protocols for MANETs discover a route when a source needs to communicate with a destination. The multi-path routing protocol discovers multiple paths during the single route discovery process. These multiple paths can be used for load spreading or as backup routes when the primary route fails [2].

AOMDV is a multi-path extension of AODV. In AODV when a source wants to communicate to a destination it initiates a route discovery process by flooding a Route Request (RREQ) packet for destination through the network. Duplicate RREQs are recognized, and discarded, using unique sequence numbers. An intermediate node, receiving a non-duplicate RREQ packet, first sets up a reverse path to the source using the previous hop of the RREQ as the next hop on the reverse path. If a valid route to the destination is available in its routing table, then the intermediate node generates a route reply (RREP) packet, otherwise the RREQ is rebroadcast. When the destination receives a non-duplicate RREQ, it also generates RREP. The RREP is routed back to the source via the reverse path. A node updates its routing information and propagates the RREP upon receiving further RREPs only if a RREP contains either a larger destination sequence number (fresh) or a shorter route is found.
Like AODV, AOMDV is based on distance vector concept and uses hop by hop routing approach. Moreover, AOMDV also finds routes on demand using a route discovery procedure. Unlike AODV, AOMDV finds multiple routes in a single route discovery procedure. In AODV all duplicate RREQs are discarded whereas AOMDV look for an opportunity of getting an alternate route with each duplicate RREQ. In AOMDV, RREQ propagation from the source towards the destination establishes multiple reverse paths both at intermediate nodes as well as the destination. Multiple RREPs traverse these reverse paths back, to form multiple forward paths to the destination at the source and intermediate nodes. AOMDV also provides intermediate nodes with alternate paths as they are found to be useful in reducing route discovery frequency. The core of the AOMDV protocol lies in ensuring that multiple paths discovered are loop free and disjoint; and in efficiently finding such paths using a flood-based route discovery. AOMDV route update rules, applied locally at each node, play a key role in maintaining loop-freedom and disjoint-ness properties. [1, 8$11]$.

AOMDV is usually compared with AODV. In this paper, AOMDV performance is investigated with respect to CBR and TCP traffic.

\section{SIMULATION ENVIRONMENT}

We used network simulator-2 (ns-2) version 2.34 [12] for the simulation and the 802.11 MAC protocol for shared access to wireless channels. AOMDV protocol is simulated with CBR and TCP traffic. The simulation parameters used are shown in Table I. The size of the topology was set in a 1000x1000 grid. Multiple sources and destinations were used. The traffic types analyzed are CBR and TCP. Random Waypoint mobility model is used for node mobility. Packet transmission rate is modified. We have analyzed the performance of AOMDV with CBR and TCP traffic types at different rate of packet transmission. Also different numbers of sources and destinations are used. We used 100 nodes having 50 connections for both CBR and TCP traffic. The simulation run time was set to $200 \mathrm{~s}$. Data packet size was set to 512 bytes. We have measured the following: throughput, Drop Packet Ratio (DPR), Average Delay (Avg Delay), route discovery frequency and routing overhead.

\section{RESULT AND ANALYSIS}

Here we study the effect of data rate on the performance of AOMDV. The mean node speed is kept to 5 and the number of connections to 50 . The data packet generation rate is increased at each connection from 1 to 5 packets/s.

For each packet rate the simulation is run for $200 \mathrm{~s}$. Figures 1 to 5 show the analysis of AOMDV with respect to the packet rate. Figures 6 to 8 give the analysis of AOMDV traffic with respect to average delay. It is evident from the following graphs that AOMDV performance degrades with the increase in packet rate. 


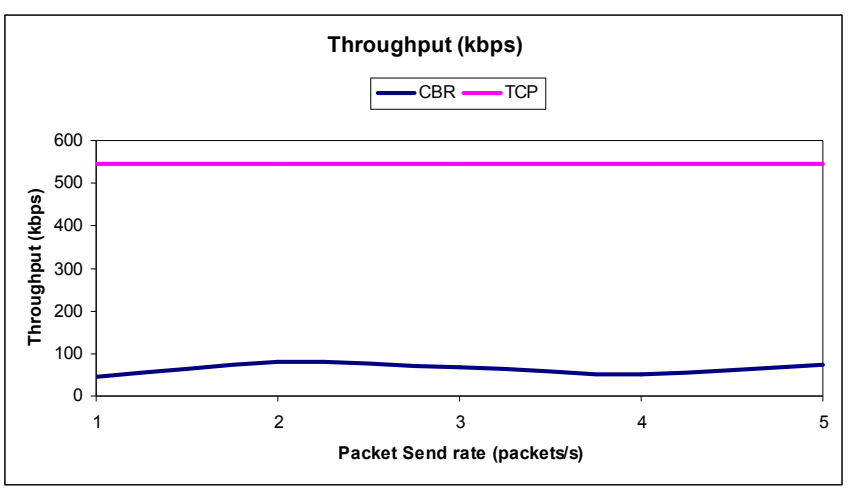

Fig. 1. Throughput vs. packet send rate

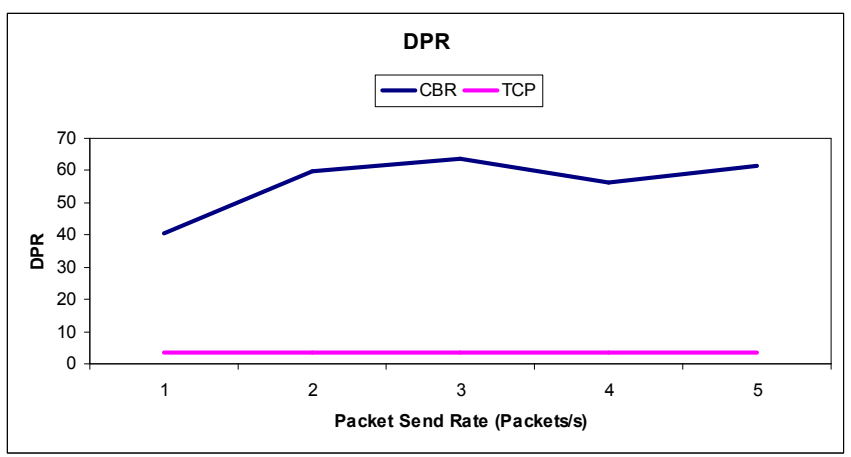

Fig. 2. DPR vs. packet send rate

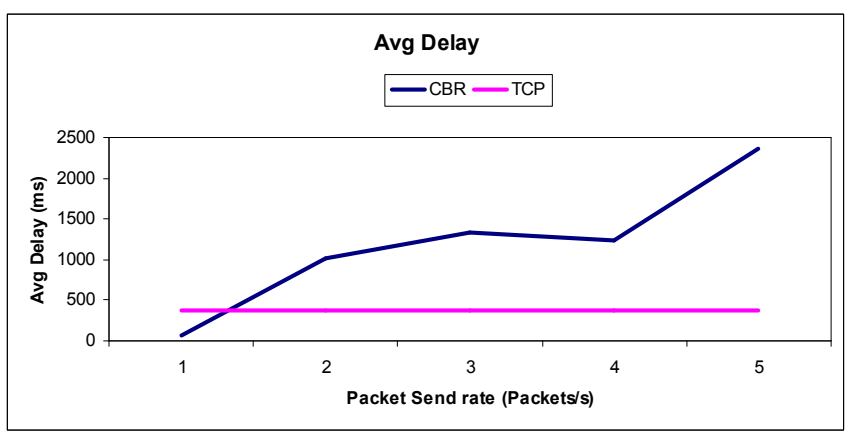

Fig. 3. Avg Delay vs. packet send rate

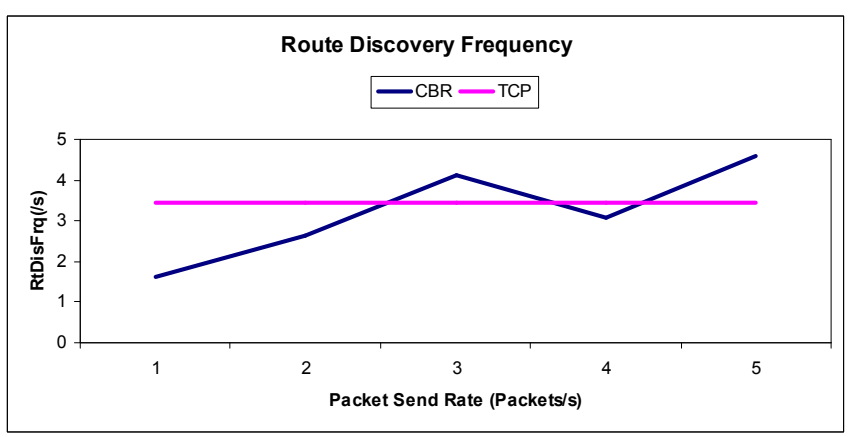

Fig. 4. Route Discovery Frequency vs. packet send rate

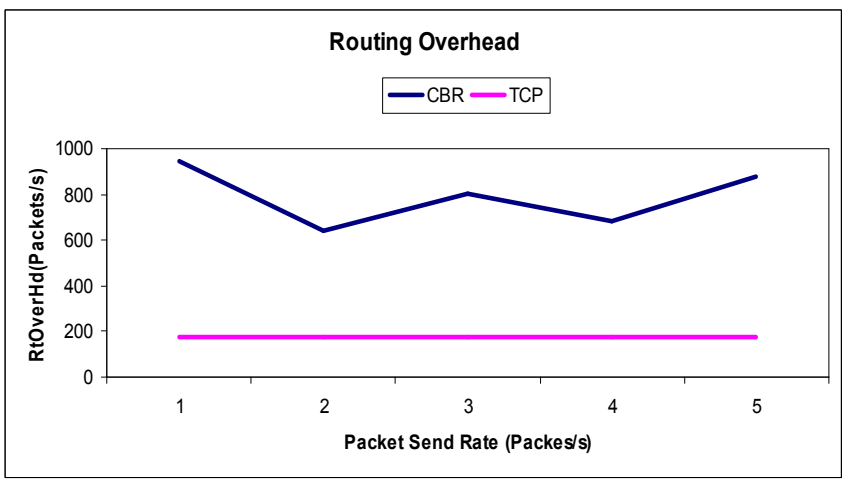

Fig. 5. Routing Overhead vs. packet send rate

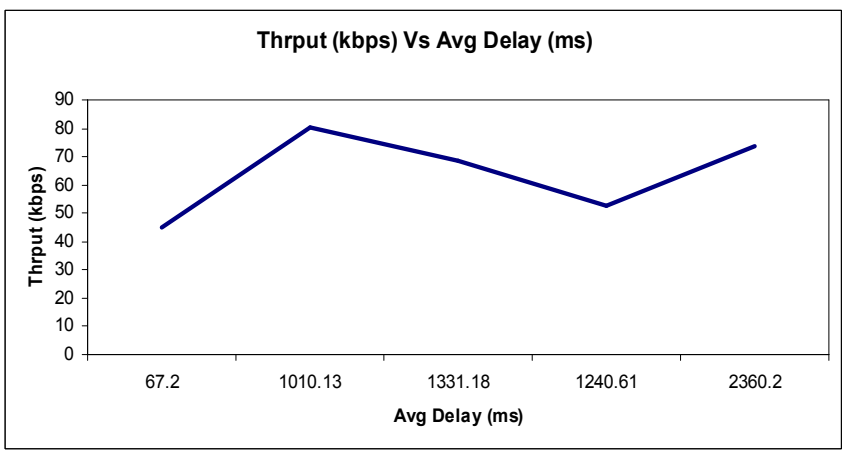

Fig. 6. Throughput vs. Avg Delay

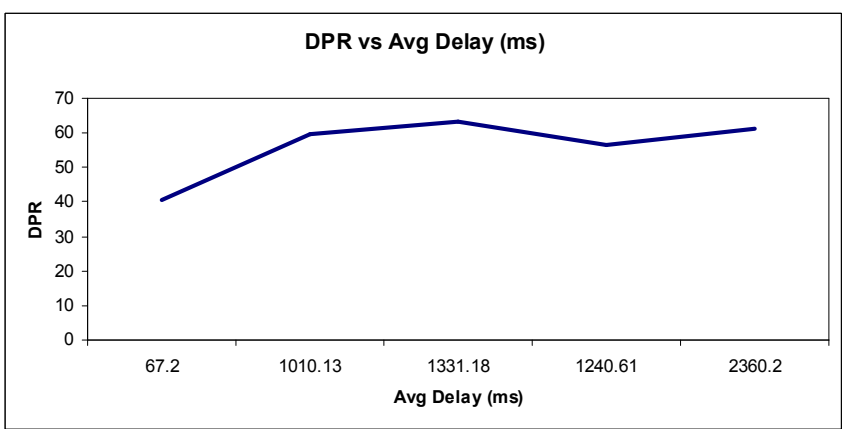

Fig. 7. DPR vs. Avg Delay

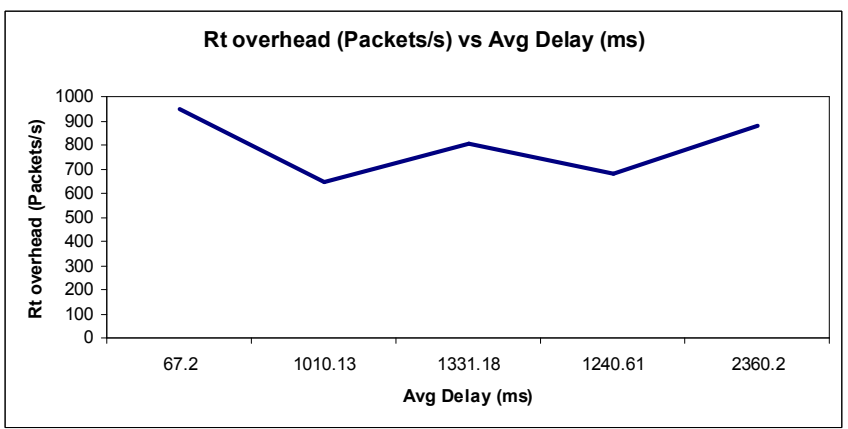

Fig. 8. Routing overhead vs Avg Delay 
TABLE I. MULATION PARAMETERS

\begin{tabular}{|c|c|}
\hline Parameters & Values \\
\hline Topology size & $1000 \times 1000$ \\
\hline No. of Nodes & 100 \\
\hline No. of Sources & Multiple \\
\hline No. of Destinations & Multiple \\
\hline Packet size & 512 bytes \\
\hline MAC protocol & IEEE 802.11 \\
\hline Simulation time & 200 s \\
\hline Traffic Types & CBR/TCP \\
\hline Simulation run & 200 s for each packet rate \\
\hline
\end{tabular}

\section{CONCLUSION AND FUTURE WORK}

In this paper we discus AOMDV routing protocol, a multipath extension to AODV. We also discuss different QoS issues for Mobile ad hoc networks. We have analyzed different QoS issues of AOMDV with varying data packet generation rate. From the simulation results we concluded that with increase in packet rate AOMDV performance degrades for CBR traffic whereas for TCP traffic it is consistent. In future work, AOMDV will be analyzed with respect to the mean node speed, which is kept constant in this paper, and to the number of connections.

\section{REFERENCES}

[1] M. K. Marina, S. R. Das, "Ad hoc on-demand multipath distance vector routing”, Wirel. Commun. Mob. Comput., Vol. 6, pp. 969-988, 2006

[2] V. C. Patil, R. V. Biradar, R. R. Mudholkar, S. R. Sawant, "On-demand multipath routing protocols for mobile ad hoc networks issues and comparison", International Journal of Wireless Communication and Simulation, Vol. 2, No 1, pp. 21-38, 2010

[3] L. Hanzo II, R. Tafazolli, "A Survey of QoS routing solutions for mobile ad hoc networks", IEEE Communications Surveys \& Tutorials, Vol. 9, No. 2, pp. 50-70, 2007
[4] Y. Lu, Y. Zhong, B. Bhargava, "Packet Loss in mobile ad hoc networks", Technical Report, Dept. of Comp. Sci, Purdue Uni., TR 03009,2003

[5] J. Liu, F. Li, "An improvement of AODV protocol based on reliable delivery in mobile ad hoc networks", 5th International Conference on Information Assurance and Security, Vol. 1, pp. 507-510, 2009

[6] S. Malini, E. Kannan, A. Valarmathi, C. Daniel Sundar Rajan, "Traffic based energy consumption analysis of AOMDV protocol in a mobile ad hoc network", International Journal of Computer Applications, Vol. 14, No. 6, pp. 43-46, 2011

[7] A. R. Sangi, J. Liu, Z. Liu, "Performance comparison of single and multi-path routing protocol in MANET with selfish behaviours", World Academy of Science, Engineering and Technology, Vol. 41, pp. 828832,2010

[8] M. K. Marina, S. R. Das, "On-demand multipath distance vector routing in ad hoc networks", IEEE 9th International Conference on Network Protocols (ICNP), Vol. 1, pp. 14-23, 2001

[9] M. T. Toussaint, "Multipath routing in mobile ad hoc networks", Report, DTC. 5966 Freeband/CACTUS Project, 2003

[10] C. E. Perkins, E. M. Royer, "Ad hoc on-demand distance vector routing”, IEEE 2nd Workshop on Mobile Computing Systems and Applications (WMCSA), Vol. 1, pp. 90-100, 1999.

[11] V. B. Kute, M. U. Kharat, "Survey on QoS for multi-path routing protocols in mobile ad-hoc networks", 3rd International Conference on Machine Learning and Computing (ICMLC 2011), Vol. 4, pp. 524-528, 2011

[12] K. Fall, K. Varadhan, The Ns Manual, http://www.isi.edu/nsnam/ns/nsdocumentation.html, last accessed: Jan 6, 2009 